\title{
Oral anti-diabetics for gestational diabetes mellitus - where do we stand?
}

\author{
Delia Cudalba', Corina Gica', Radu Botezatu',2, Nicolae Gica ${ }^{1,2}$, Anca Marina Ciobanu', \\ Gheorghe Peltecu ${ }^{1,2}$, Anca Maria Panaitescu, ${ }^{1,2}$ \\ ${ }^{1}$ Filantropia Clinical Hospital, Bucharest, Romania \\ 2"Carol Davila" University of Medicine and Pharmacy, Bucharest, Romania
}

\begin{abstract}
Background. Oral anti-diabetics in the treatment of gestational diabetes mellitus have had different approaches among time in medical guidelines. The interest in this pregnancy-associated disease is due to the dramatic increase of rates of gestational diabetes but also to the significantly higher short-term and longterm implications for mother and off-spring. This article aims to review the literature and guidelines on current recommendations regarding oral therapy in gestational diabetes mellitus.

Methods. A systematic literature electronic search for reviews and guidelines was undertaken using Pub Med and the official websites of Diabetes and Obstetrics and Gynecology associations.

Conclusions. Whereas oral anti-diabetics are an attractive alternative approach to insulin, in the last guidelines published by the societies of Obstetrics and Gynecology and Diabetes a new trend is noticeable subtracting oral anti-diabetics as the first option because of the lack in high-quality data on long-term safety, higher rate of treatment failure and pharmacokinetics.
\end{abstract}

Keywords: gestational diabetes mellitus, oral anti-diabetics, insulin, metformin, glibenclamide

\section{INTRODUCTION}

\section{Definition}

Gestational diabetes mellitus (GDM) is one of the most common endocrine diseases that occurs during pregnancy and has been commonly defined as "glucose intolerance or hyperglycemia of variable degree that occurs or is firstly recognized during pregnancy" and that usually withdraws after birth [1].

The constant increase in maternal age, less active lifestyle, obesity rate and other behavioral risk factors rise the prevalence of this type of carbohydrate intolerance.

The interest in this pregnancy-associated disease is due to the dramatic increase of rates of GDM but also to the significantly higher short-term and long-term implications for mother and off-spring.

\section{Prevalence}

International Diabetes Federation (IDF) estimated that in 2019223 million women were affected by diabetes and the projection for 2045 would be an increase up to 343 million. When it comes to pregnancy, IDF studies show that 20 million (16\%) of live births were affected by an impairment of glucose tolerance and GDM was diagnosed in $84 \%$ of cases (approximately 1 in 6 births). Centers for Disease Control and Prevention (CDC) approximation for the United States was that $6 \%$ to $9 \%$ of pregnant women will develop gestational diabetes. From 2000 to 2010, the percentage of pregnancies that associated gestational diabetes increased by $56 \%$ [2]. For Europe some studies estimated in 2016 that the prevalence was between 5.4 and 5.8\% $[2,3]$. Hispanic, African, Asian and American women have greater risk of developing GDM [4]. 


\section{METHODS}

A systematic literature electronic search for reviews and guidelines was undertaken using Pub Med and the official websites of Diabetes and Obstetrics and Gynecology associations. Search words were "gestational diabetes mellitus", "oral anti-diabetics" and "gestational diabetes mellitus" combined with "diagnosis", "treatment", "management", "insulin", "metformin". Publications were selected based on accessibility to full paper article, quality evaluation, publication year. The publications used are mentioned in References section. The guidelines selected were the last published, comparing the recommendations with those in the previous guidelines.

\section{RESULTS}

\section{Diagnosis}

Screening for diabetes in pregnancy should be firstly an early screening for undiagnosed type 2 diabetes recommended for overweight and obese women with additional risk factors (certain ethnicities, family history of GDM or type 2 diabetes, a previous large baby, older age). It is preferably done at the beginning of the prenatal care using standard diagnostic criteria [1].

Screening for GDM is done at 24-28 weeks of gestation in pregnant women not previously diagnosed with diabetes. The American Diabetes Association (ADA) has recommended two options, even each organization has its preferences and so far no strategy proved to be optimal.

The "one-step" 75-g oral glucose tolerance test (OGTT) was recommended in 2010 by the International Association of Diabetes and Pregnancy Study Group, but even if they are still promoting it, ADA admitted in 2017 that there is no sufficient evidence supporting those criteria, as these cutoffs would increase the prevalence of GDM [5]. It is based on 75-g OGTT with plasma glucose measurement after fasting for at least 8 hours and after 1 and 2 hours. Gestational diabetes mellitus is diagnosed when the fasting result is $\geq 92 \mathrm{mg} / \mathrm{dl}(5.1 \mathrm{mmol} / \mathrm{l})$, 1 hour $\geq 180 \mathrm{mg} / \mathrm{dl}(10.0 \mathrm{mmol} / \mathrm{l})$ and 2 hours $\geq 153 \mathrm{mg} / \mathrm{dl}$ ( $8.5 \mathrm{mmol} / \mathrm{l})$ [4].

The older "two-step" approach, used since 1973 and supported by the American College of Obstetricians and Gynecologists (ACOG) is a $50-\mathrm{g}$ OGTT (non-fasting), measuring plasma glucose level after 1 hour and performing a fasting $100-\mathrm{g}$ OGTT if the values exceed $130 \mathrm{mg} / \mathrm{dl}, 135 \mathrm{mg} / \mathrm{dl}$ or
$140 \mathrm{mg} / \mathrm{dl}(7.2 \mathrm{mmol} / 1,7.5 \mathrm{mmol} / 1$, or $7.8 \mathrm{mmol} / 1)$. Gestational diabetes mellitus is diagnosed if two or more of the following OGTT plasma glucose levels are $\geq 95 \mathrm{mg} / \mathrm{dl}(5.3 \mathrm{mmol} / \mathrm{l})$ for fasting, $\geq 180 \mathrm{mg} /$ $\mathrm{dl}(10.0 \mathrm{mmol} / \mathrm{l})$ at $1 \mathrm{hour}, \geq 155 \mathrm{mg} / \mathrm{dl}(8.6 \mathrm{mmol} / \mathrm{l})$ at 2 hours or $\geq 140 \mathrm{mg} / \mathrm{dl}(7.8 \mathrm{mmol} / \mathrm{l})$ at 3 hours $[4,6]$.

\section{The impact of GDM}

The purpose of treating GDM is reducing the maternal impact as well as the neonatal and infant outcomes. "Hyperglycemia and Adverse Pregnancy Outcomes" (HAPO), a large-scale cohort study comprising 23,000 participants, published in 2008, proposed to clarify the relationship between the degree of maternal glucose intolerance and the adverse outcomes, beginning from the situation caused by the lack in information regarding "whether maternal hyperglycemia less severe than that in diabetes mellitus is associated with increased risks of adverse pregnancy outcomes". Among another conclusions, it proved a constant relationship between the degree of hyperglycemia and the maternal and neonatal risk [7].

The acute maternal outcomes are urinary tract infections and vaginal infections of higher incidence leading to an increased rate of premature delivery. Women affected by GDM have higher rates of uterine ruptures, perineal lacerations, placental abruption, post-partum hemorrhage, infection and preeclampsia. They are more likely to give birth by caesarean section or by having their labor induced. There are also long-term maternal outcomes as the recurrence of diabetes and metabolic dysfunction in later life, consequences that associate higher risk for coronary heart disease and stroke $[8,9]$.

The outcomes, when assessing the results of GDM on the newborn, are diabetic fetopathy (hypoglycemia, hypocalcemia, hypomagnesemia, hyperbilirubinemia, respiratory disorders), macrosomia, shoulder dystocia, bone fracture, nerve palsy and the possible metabolic dysfunction in childhood and adult life [8,9].

\section{Management of GDM}

The management of GDM begins with medical nutrition therapy, trying to maintain euglycemia while providing enough nutritional support for pregnancy. Studies show that $70-85 \%$ of women can obtain glycemic control under individualized nutrition counseling and physical activity. If exercise and diet fail to control the glycemic level, the next step is the pharmacological approach [10]. 
Current pharmacological options in GDM treatment are subcutaneous insulin and oral anti-diabetics. The guidelines regarding GDM medication has been constantly changing among time. The first approach was diet and insulin. It is reported that before 2000 less than $1 \%$ of women used oral anti-diabetics, percentage that reached $7.4 \%$ in 2001 and $64.5 \%$ in 2011 , with glyburide as firstline agent in $57 \%$ of cases and metformin in only $4 \%[10]$.

In 2013 ACOG stated that insulin and oral hypoglycemic agents can be both used as first-line agents but the 2017 ACOG Practice Bulletin recommended insulin as first choice and metformin as a second-line agent, recommendation still supported by ACOG in 2018 [4,11,12].

In 2018 ADA stated that insulin is the preferred medication and metformin and glyburide "may be used", mentioning that they both cross the placenta and there is a lack of long-term safety data. Since 2019 ADA's opinion regarding the use of oral anti-diabetics is that they "should not be used as first-line agents" $[1,13]$.

The studies published in the last decade that tried to compare each pharmacological option took into consideration some outcome measures: maternal (pregnancy-induced hypertension, eclampsia, cesarean section, the use of additional pharmacotherapy, glycemic control) and neonatal (macrosomia, hypoglycemia, respiratory distress syndrome) [8].

The main criteria used in those comparisons are the glycemic control, the maternal and neonatal side effects and the possible secondary positive effects [9].

The most studied agents are metformin and glibenclamide. Oral anti-diabetics are an attractive alternative approach to insulin because of the facility of administration, acceptability and lower costs, advantages that could increase the patient medication adherence. Their pitfalls are that they both cross the placenta, there is a lack in high-quality data on long-term safety and a higher rate of treatment failure.

\section{Metformin}

Metformin is an oral agent of the biguanide class that acts by reducing hepatic glucose gluconeogenesis, decreasing intestinal absorption of glucose and increasing peripheral glucose uptake and utilization, thus enhancing insulin sensitivity [14]. The dosage starts at $500 \mathrm{mg}$ and can reach 3.000 $\mathrm{mg}$ per day in two or three divided doses [4]. Metformin should be avoided in patients with impaired renal function, heart failure and severe liver disease [14].

Since insulin remains the first-line recommendation, the efficacy of metformin is mainly compared with it. In ADA 2020 guideline, metformin, compared to insulin was associated with less maternal weight gain, lower risk of neonatal hypoglycemia and better secondary effects on pregnancy induced hypertension. A short-term disadvantage would be that metformin may increase the risk of prematurity and on long-term some studies that followed the off-springs proved that the exposure to metformin increased BMI and the rate of obesity [15]. An indisputable fact about metformin pharmacokinetics is that it crosses the placenta and reaches as high or higher levels than maternal ones. A contraindication in the use of metformin is maternal hypertension, preeclampsia and possible intrauterine growth restriction [16]. The ACOG Practice Bulletin 2018 mentions a few studies, but even some of them concluded that metformin and insulin have similar outcomes, there is a conflict generated by the quality of the studies. Another conclusion is that between $26 \%$ and $46 \%$ of the women who used metformin alone needed additional insulin [4].

The lack of long-term neonatal follow-up after metformin exposure is one reason why ADA is recommending insulin as first option.

\section{Glibenclamide}

Glibenclamide, also known as glyburide, is second generation sulfonylureas administered alone or as part of combination therapy with metformin, acting by stimulating the insulin secretion. Concentrations of glyburide in umbilical cord plasma are $50-70 \%$ of the levels in maternal plasma [1].

Studies showed that when compared to insulin, it is associated with less maternal hypoglycemia but a higher rate neonatal hypoglycemia, macrosomia and hyperbilirubinemia, preeclampsia and stillbirth. Compared to metformin, glyburide lacks in long-term safety data. When it comes to glycemic control, 4-16\% of women treated with glibenclamide required additional insulin $[1,4]$.

When comparing metformin and glibenclamide, in terms of maternal weight gain and macrosomia, metformin had better outcomes [15].

\section{DISCUSSION}

In the last guidelines published by the societies of Obstetrics \& Gynecology and Diabetes a new 
trend is noticeable subtracting oral anti-diabetics as the first option.

The last guideline published by the American Diabetes Association (ADA) on January 2020 states that the first-line agent should be insulin and when metformin is used in the treatment of polycystic ovary syndrome it shouldn't be used longer than the first trimester of pregnancy [1].

The American College of Obstetricians and Gynecologists lines up with ADA recommendation, but accepts that oral anti-diabetic therapy could be used when the use of insulin is a problem in terms of the safety of administration, the costs or the decline of the patient, as long as the limitations of metformin and glyburide are explained [4].

National diabetes societies from Germany, Austria and Switzerland published in 2018 a common guideline in which the next step, if physical activity and dietary counselling cannot maintain normal blood glucose levels, is administration of insulin. Metformin use is accepted in case of insulin resistance, taking into account the contraindications and dosage. Glibenclamide and another oral anti-diabetic are not allowed to be prescribed due to lack of data [8].

In 2017, Cochrane published a review including 11 studies (1,487 women) which purposed to compare oral anti-diabetic pharmacological therapies. The authors' conclusion was that there is insuffi-

\section{REFERENCES}

1. American Diabetes Association. 2. Classification and Diagnosis of Diabetes: Standards of Medical Care in Diabetes-2020. Diabetes Care. 2020;43(Suppl 1):S14-S31.

2. Dawn MC, Josie MME, Eades CE. Prevalence of gestational diabetes mellitus in Europe: A meta-analysis. Diabetes Research and Clinical Practice (Elsevier) 2017;129: 173-181.

3. Zhang C, Zhu Y. Prevalence of Gestational Diabetes and Risk of Progression to Type 2 Diabetes: a Global Perspective. Current Diab Rep. 2016;16(1):7.

4. The American College of Obstetricians and Gynecologists. Gestational Diabetes Mellitus. ACOG Practice Bulletin 2018 No 190;131(2):49-59.

5. Metzger BE, Gabbe SG, Persson B et al. International Association of Diabetes and Pregnancy Study Groups recommendations on the diagnosis and classification of hyperglycemia in pregnancy. Diabetes Care. 2010;33(3):676-680.

6. O'Sullivan JB, Mahan CM, Charles D et al. Screening criteria for high-risk gestational diabetic patients. Am J Obstet Gynecol. 1973; 11(7):895-900.

7. HAPO Study Cooperative Research Group. Hyperglycemia and Adverse Pregnancy Outcome (HAPO) Study: associations with neonatal anthropometrics. Diabetes. 2009;58(2):453-9.

8. Gembruch U, Kainer F, Ute M et al. Gestational Diabetes Mellitus (GDM) - Diagnosis, Treatment and Follow-Up. Guideline of the DDG and DGGG. Geburtshilfe Frauenheilkd. 2018;78(12):1219-1231. cient high-quality data in order to draw conclusions over the non-inferiority of one oral anti-diabetic drug [17].

\section{CONCLUSIONS}

Oral anti-diabetic medication is not currently recommended as first-line pharmacological approach, but here are still some discrepancies between guidelines. Uniformity of guidelines on the management of GDM is highly inconstant and needs to be improved and clarified.

For the time being the medical specialties that deal with GDM are obstetrics and gynecology and diabetology. The obstetrician that is monitoring the pregnancy screens for GDM, diagnoses it and refers the future mother to a diabetologist which manages the disease. It is necessary that the recommendations provided by both of the medical specialties to be convergent. The quality of the treatment is then assessed not only by the obstetrician, neonatologist and pediatrician, because the outcomes can be long-term and trans-generational.

The concern of oral anti-diabetics in GDM require further research for the well-being of both mother and offspring.

\section{Acknowledgement.}

All authors equally contributed to this article.

Conflict of interest: none declared Financial support: none declared

9. Brown J, Martis R, Hughes B et al. Oral anti-diabetic pharmacological therapies for the treatment of women with gestational diabetes. Cochrane Database of Systematic Reviews. 2017;1(CD011967):1-83.

10. Castillo C, Boggess K, Sturmer T et al. Trends in glyburide compared with insulin use for gestational diabetes treatment in the United States: 2000-2011. Obstet Gynecol. 2014;123(6):1177-1184.

11. American College of Obstetricians and Gynecologists. Practice Bulletin No. 137: Gestational diabetes mellitus. Obstet Gynecol. 2013;122(2):406-416.

12. American College of Obstetricians and Gynecologists. Practice Bulletin No 180: Gestational diabetes mellitus. Obstet Gynecol. 2017;130(1):e17-e37.

13. American Diabetes Association, 13. Management of Diabetes in Pregnancy. Diabetes Care. 2018;41 (Suppl 1):S137-S143.

14. DrugBank.ca [Online]. Created on June 13, 2005/ Updated on August 10, 2020. Available from: www.drugbank.ca/drugs/DB0033.

15. Balsells M, García-Patterson A, Solà I et al. Glibenclamide, metformin, and insulin for the treatment of gestational diabetes: A systematic review and meta-analysis. BMJ. 2015;350(102):1-12.

16. Barbour LA, Feig DS. Metformin for gestational diabetes mellitus: Progeny, perspective, and a personalized approach. Diabetes Care. 2019;42(3):396-399.

17. Zhang M, Zhou Y, Zhong J et al. Current guidelines on the management of gestational diabetes mellitus: A content analysis and appraisal. BMC Pregnancy and Childbirth. 2019;19(200):1-15 